\title{
Estimation of Texels for Regular Mosaics Using Model-Based Interaction Maps
}

\author{
Georgy Gimel'farb* \\ CITR, Department of Computer Science, Tamaki Campus, University of Auckland \\ Private Bag 92019, Auckland 1, New Zealand \\ g.gimelfarb@auckland.ac.nz
}

\begin{abstract}
Spatially homogeneous regular mosaics are image textures formed as a tiling, each tile replicating the same texel. Assuming that the tiles have no relative geometric distortions, the orientation and size of a rectangular texel can be estimated from a model-based interaction map (MBIM) derived from the Gibbs random field model of the texture. The MBIM specifies the structure of pairwise pixel interactions in a given training sample. The estimated texel allows us to quickly simulate a large-size prototype of the mosaic.
\end{abstract}

\section{Introduction}

Image texture is usually thought of as being formed by spatial replication of certain pixel neighbourhoods called texels [6]. Replicas of each texel may differ providing their visual similarity is not effected. We restrict our consideration to a limited number of regular textures such as translation invariant tilings, or mosaics formed with a single rectangular texel. The texel-based description holds considerable promise for fast simulation of large-size samples of these textures.

A significant advance in realistic texture simulation has been made recently by approximating particular pixel neighbourhoods of a given training sample with the neighbourhoods of the simulated image $[2,3,4,5,7,9,10,11]$. The chosen neighbourhoods preserve the deterministic spatial structure of signal interactions in the training sample. The approximation extrapolates the training structure to images of other size and provides random deviations from the training sample.

In most cases the pixel neighbourhoods are implicitly accounted for by using certain spatial features of a multi-resolution image representation, e.g. the top-to-bottom signal vectors along a Laplacian or steerable wavelet image pyramid $[2,10]$, or the explicit neighbourhoods such as squares $7 \times 7$ pixels are chosen in a heuristic way to take account of conditional relative frequency distributions of the multi-resolution signals over these small close-range neighbourhoods [9]. The explicit heuristic neighbourhoods are used also in the non-parametric texture sampling $[3,7]$ where each new pixel or small rectangular patch is chosen equiprobably from the pixels or patches having closely similar neighbourhoods

\footnotetext{
* This work was supported by the University of Auckland Research Committee grants 9343/3414113 and 9393/3600529 and the Marsden Fund grant UOA122.
} 
in the training sample or in the already simulated part of the goal image. The most characteristic explicit pixel neighbourhood describing a single-resolution texture is analytically estimated for the Gibbs random field models with multiple pairwise pixel interactions $[4,5,11]$.

Although these techniques are efficient in simulating different natural textures, most of them involve too considerable amounts of computations per pixel to form large-size samples of the texture. The patch-based non-parametric sampling [7] is much faster than other approaches but the quality of simulation depends on the heuristic choice of the patches and their neighbourhoods.

In this paper we consider possibilities of estimating texels for certain regular mosaics using the explicit spatial structure of multiple pairwise pixel interactions in the Gibbs random field model found for the training sample. Then the largesize prototypes of the mosaic can be obtained very fast by replicating the texel.

\section{Model-Based Interaction Maps and Texels}

Let $g: \mathbf{R} \rightarrow \mathbf{Q}$ where $\mathbf{R}$ and $\mathbf{Q}$ denote an arithmetic lattice and a finite set of image signals, respectively, be a digital image. Let the set $\mathbf{A}$ of inter-pixel shifts specify a translation invariant neighbourhood $\mathbf{N}_{i, \mathbf{A}}=\{(i+a): a \in \mathbf{A}\}$ of each pixel $i \in \mathbf{R}$. As shown in [4], the approximate partial Gibbs energy $E_{a, 0}(\hat{g})$ of interactions over the family of the similar pixel pairs $\mathbf{C}_{a}=\{(i, i+a): i \in \mathbf{R}\}$ in a given training sample $\hat{g}$ is easily obtained from the relative frequency distribution $\mathbf{F}_{a}(\hat{g})=\left\{F_{a}(q, s \mid \hat{g}): q, s \in \mathbf{Q}\right\}$ of the signal co-occurrences $\left(\hat{g}_{i}=q, \hat{g}_{i+a}=s\right)$ :

$$
E_{a, 0}(\hat{g})=\sum_{(q, s) \in \mathbf{Q}^{2}} F_{a}(q, s \mid \hat{g})\left(F_{a}(q, s \mid \hat{g})-\frac{1}{|\mathbf{Q}|^{2}}\right)
$$

The interactions specified by a large search set $\mathbf{W}$ of the inter-pixel shifts $a$ can be ranked by their partial energies, and the characteristic interaction structure $\mathbf{A}$ is estimated by a parallel or sequential selection of the top-rank energies $[4,5,11]$.

Figure 1 shows training samples $128 \times 128$ of the natural image textures from $[1,8]$ and the scaled images $81 \times 81$ of their model-based interaction maps (MBIM) $\mathbf{E}(\hat{g})=\left\{E_{a, 0}(\hat{g}): a \in \mathbf{W}\right\}$ showing the structure of pairwise pixel interactions. Every spatial position $a \equiv(x, y)$ of the depicted MBIM indicates the partial interaction energy $E_{a, 0}(\hat{g})$ for the inter-pixel shift $a \in \mathbf{W}$, the diametrically opposite shifts $(x, y)$ and $(-x,-y)$ representing the same family $\mathbf{C}_{a}$. In these examples the set $\mathbf{W}$ contains all the relative inter-pixel $x$ - and $y$-shifts in the range $[-40,40]$ specifying $|\mathbf{W}|=3280$ families of the pixel pairs. By the chosen greyscale coding, the larger the energy, the darker the dot.

As is easy to see in Fig. 1, the MBIMs reflect to a large extent the repetitive pattern of each texture. Replicate spatial clusters of the larger Gibbs energies indicate periodic parts of the texture, and relative positions of and pitches between the clusters relate to the overall rectangular or hexagonal shapes, spatial arrangement, and orientation of the parts. The choice of a single texel is not unique because the same MBIM defines many equivalent periodic partitions with different biases with respect to the lattice. 
D1
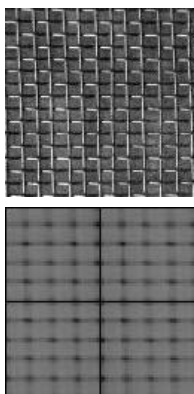

D34

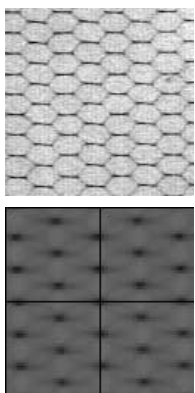

D65

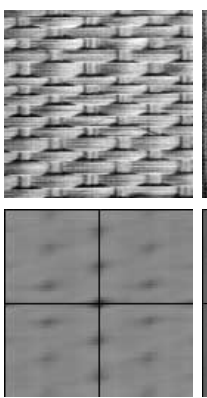

D101, rot. $20^{\circ}$
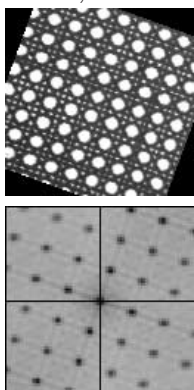

D6
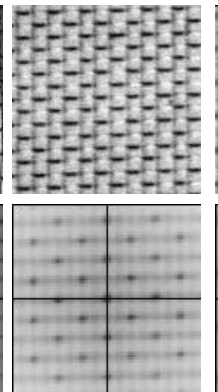

D34, rot. $-5^{\circ}$ D34, rot. $-20^{\circ}$
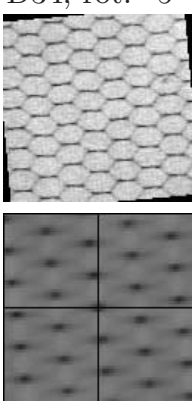

D83
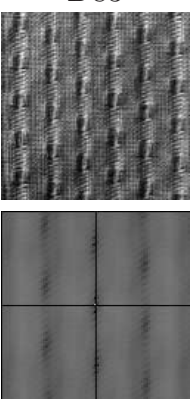

D102

$0: 0: 0$ $0-0 \cdot 0$ $000: 0$ $0-000$ $0: 0$

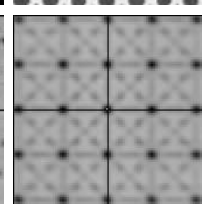

D14
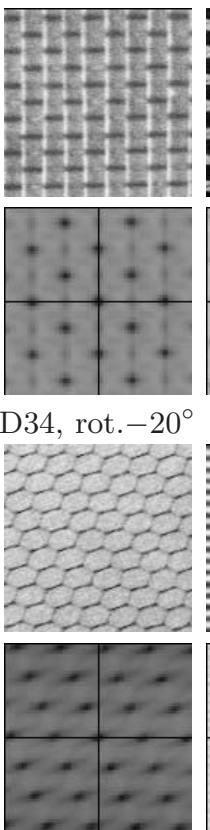

D95
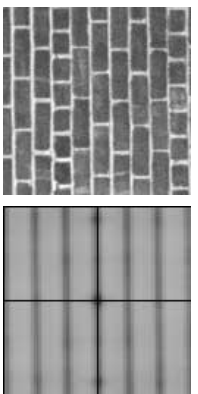

Fabric0008
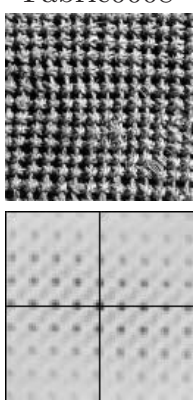

D20

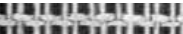

iriminitimi

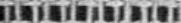

istivinginimi

riviming

Iminimini
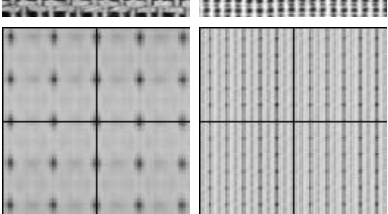

D53

D55
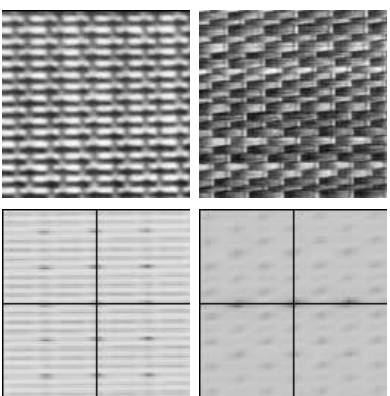

D101

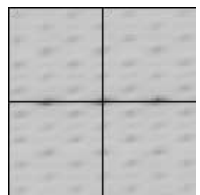

D101, rot. $5^{\circ}$

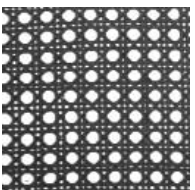

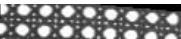

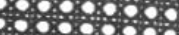
. 0090 . $0.8 \%$ 0.0.60\% 10:00:00 0.00 .0

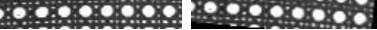

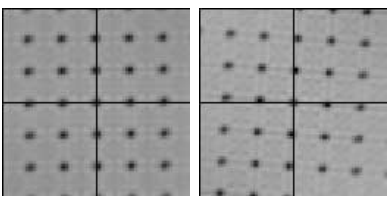

Tile0007

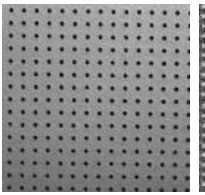

Textile0025
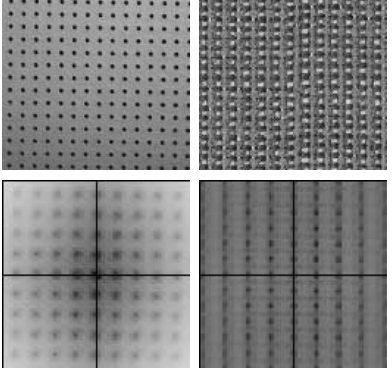

Fig. 1. Training samples and their MBIMs 
D1

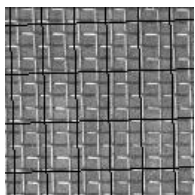

D34

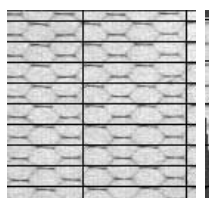

D65

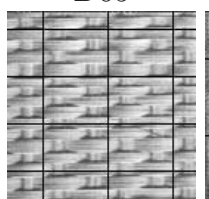

D101, rot. $20^{\circ}$
D6

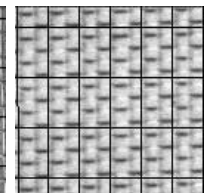

D34, rot. $-5^{\circ}$

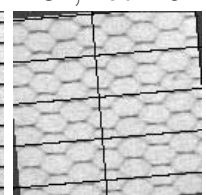

D83

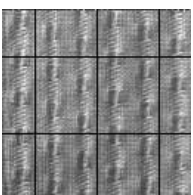

D102

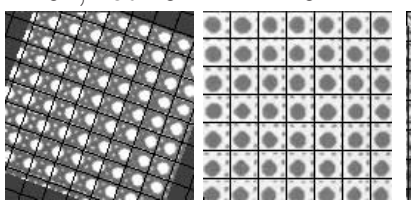

D14

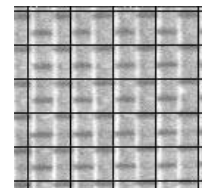

D34, rot. $-20^{\circ}$

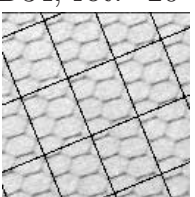

D95

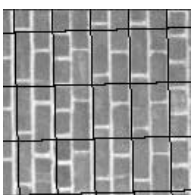

Fabric0008

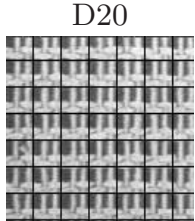

D53

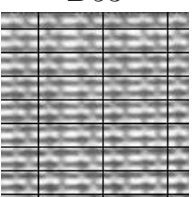

D101

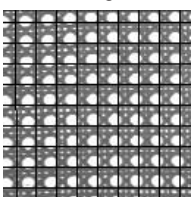

Tile0007

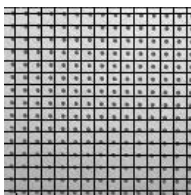

D21

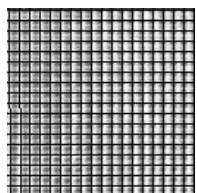

D55

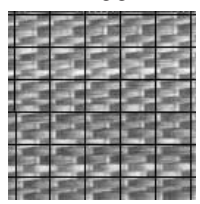

D101, rot. $5^{\circ}$

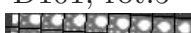

Textile0025
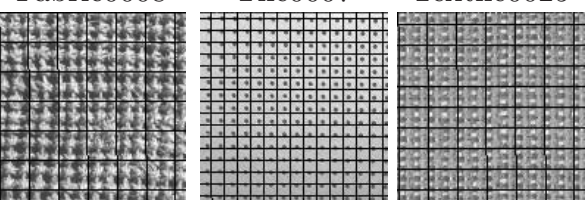

Fig. 2. Estimated partitions of the training samples

Generally it is difficult to derive a minimum-size texel from the repetitive pattern of the MBIM because some of the energy clusters may arise from the periodic fine details of the texel itself or from the secondary interactions between the distant similar parts. The shape and scale of the tiles representing the single texel as well as their photometric characteristics may also vary for different training samples and even within the same sample (e.g. D55, D95, D101, or Fabrics0008 in Fig. 1).

For simplicity, our consideration is restricted to only a rectangular texel with an arbitrary but fixed orientation and size. The central cluster of the most energetic close-range interactions in the MBIMs relates mainly to a uniform background of the image. But a repetitive pattern of the peripheral clusters (if it exists) is produced by the characteristic long-range similarities between the pixel pairs so that a single texel can be in principle estimated from the clearly defined peripheral energy clusters placed around of and closest to the central cluster.

Figure 2 demonstrates partitions of the training samples where each rectangular tile represents the texel. The partitions are estimated using a simplified heuristic approach that determines spatial clusters of the Gibbs energies by thresholding the MBIM with the threshold $E^{*}=\bar{E}+c \cdot \sigma_{E}$ where $c$ is an empiri- 
Table 1. Parameters of the rectangular texel estimated by detecting the first and second top-rank energy clusters in the MBIMs with the thresholding factor $c=2.5$ (F08, T07, T25 stand for Fabrics0008, Tile0007, and Textile0025, respectively)

\begin{tabular}{|c|c|c|c|c|c|c|c|c|c|}
\hline \multirow[t]{2}{*}{ Texture: } & \multirow[t]{2}{*}{ D1 } & \multirow[t]{2}{*}{$\overline{D 6}$} & \multirow[t]{2}{*}{ D14 } & \multirow[t]{2}{*}{ D20 } & \multirow[t]{2}{*}{$\overline{\mathrm{D} 21}$} & \multicolumn{3}{|c|}{ D34 } & \multirow[t]{2}{*}{ D53 D55 } \\
\hline & & & & & & $0^{\circ}$ & $-5^{\circ}$ & $-20^{\circ}$ & \\
\hline Texel $x$-size, pix.: & 21.5 & 21.0 & 29.0 & 19.0 & 7.0 & 70.0 & 70.26 & 34.47 & 44.024 .0 \\
\hline Texel $y$-size, pix.: & 33.02 & 34.0 & 23.0 & 18.0 & 7.0 & 14.0 & 28.24 & 42.15 & 16.022 .0 \\
\hline Orientation, ${ }^{\circ}:$ & -1.74 & 0.0 & 0.0 & 0.0 & 0.0 & 0.0 & -4.9 & -22.31 & $0.0 \quad 0.0$ \\
\hline \multirow[t]{2}{*}{ Texture: } & \multirow[t]{2}{*}{ D65 } & \multirow[t]{2}{*}{ D83 } & \multirow[t]{2}{*}{ D95 } & \multicolumn{3}{|c|}{ D101 } & \multirow[t]{2}{*}{ D102 } & \multirow[t]{2}{*}{ F08 } & \multirow[t]{2}{*}{ T07 T25 } \\
\hline & & & & $0^{\circ}$ & $5^{\circ}$ & $20^{\circ}$ & & & \\
\hline Texel $x$-size, pixels: & 44.0 & 42.0 & 25.96 & 14.0 & 15.1 & 15.2 & 19.0 & 20.0 & $9.0 \quad 20.0$ \\
\hline Texel $y$-size, pixels: & 32.0 & 52.0 & 36.76 & 14.0 & 14.04 & 13.92 & 19.0 & 20.0 & $8.0 \quad 14.0$ \\
\hline Orientation, ${ }^{\circ}:$ & 0.0 & 0.0 & -1.64 & 0.0 & 3.81 & 19.65 & 0.0 & 0.0 & 0.0 \\
\hline
\end{tabular}

cally chosen factor and $\bar{E}$ and $\sigma_{E}$ denote the mean value and standard deviation of the energies $\mathbf{E}(\hat{g})$, respectively. If the MBIM has no peripheral clusters in addition to the central cluster around the origin $a=(0,0)$, then the texture is aperiodic and has no single texel. Otherwise each peripheral cluster is described by its maximum energy and the inter-pixel shift yielding the maximum. Two clusters with the largest energy and with the second largest energy that is not occluded by the first one from the origin of the MBIM are selected to estimate the texel. The orientation is given by the smallest angular inter-pixel shift with respect to the $x$-axis of the MBIM. The size is found by projecting both inter-pixel shifts for the clusters to the Cartesian axes of the texel.

Table 1 gives parameters of the texels and partitions in Fig. 2. Changes of the thresholding factor $c$ in the range $1 \leq c \leq 3$ yield quite similar results for most of the textures used in the experiments.

This approach gives sufficiently accurate and stable estimates of the orientation angle for the rectangular and hexagonal patterns of the energy clusters in the above MBIMs. The estimates can be further refined by processing linear chains of the repetitive clusters, e.g. by finding the least scattered projections of the chains onto the coordinate axes. But the refined estimates are less stable for the hexagonal structures of the MBIMs such as for the textures D34 or D65. Generally the MBIM should be processed more in detail for finding adequate shapes, sizes, and orientations of the texels. Our approach shows only a feasibility of relating the texels to spatial periodicity of the energy clusters in the MBIMs.

Figures $3-5$ show the simulated prototypes of the size $800 \times 170$ pixels, each prototype being obtained by replicating a single tile picked arbitrarily from the partitions in Fig. 2. Of course, the singularities of the chosen tile are replicated verbatim. But all the tiles in the partition of the training sample can be jointly 

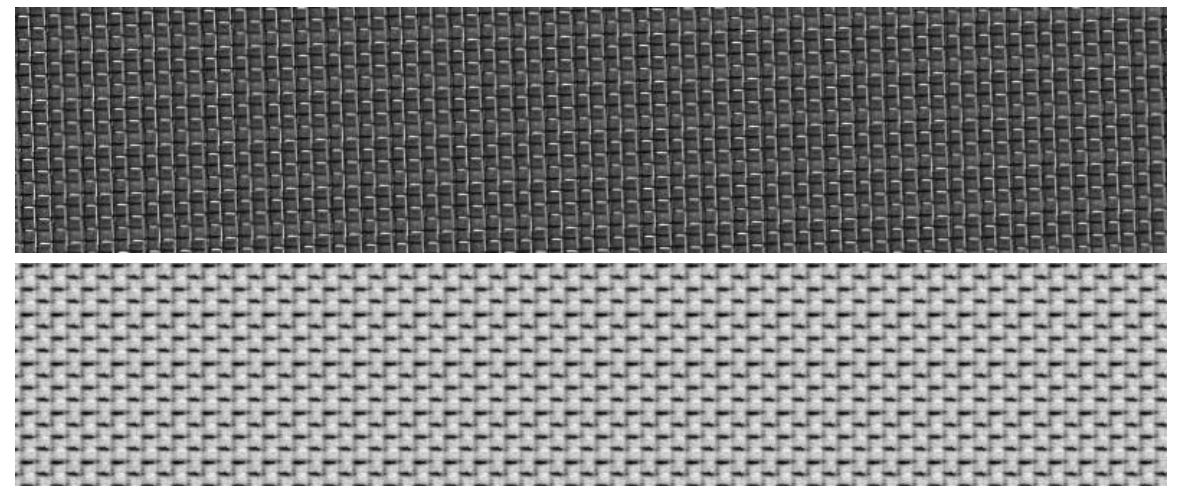

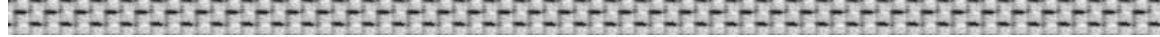

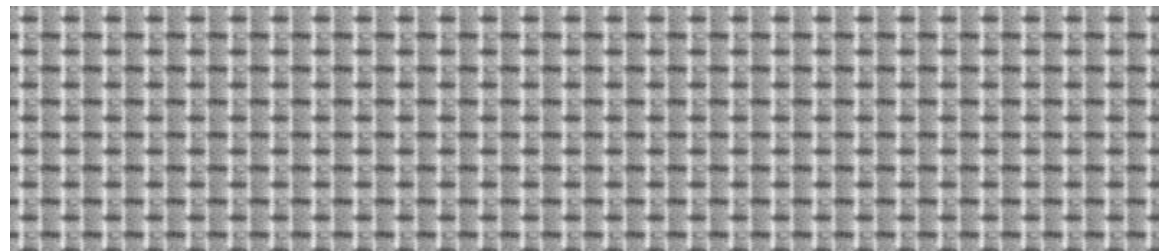

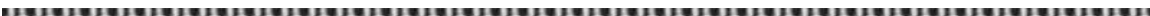

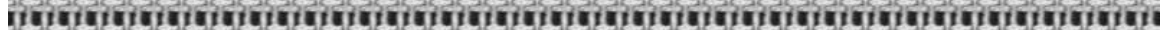

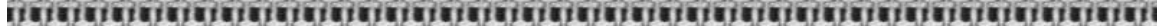

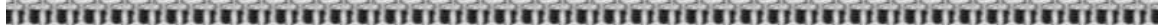
in

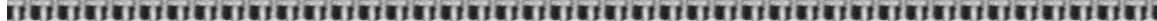

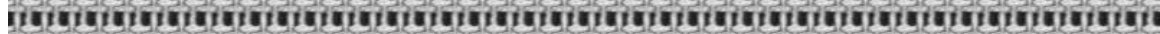

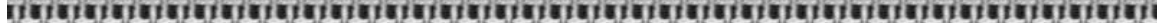

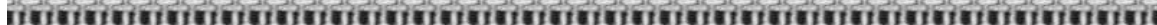

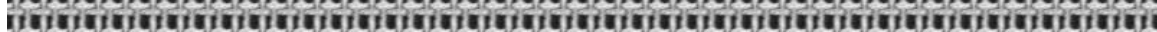

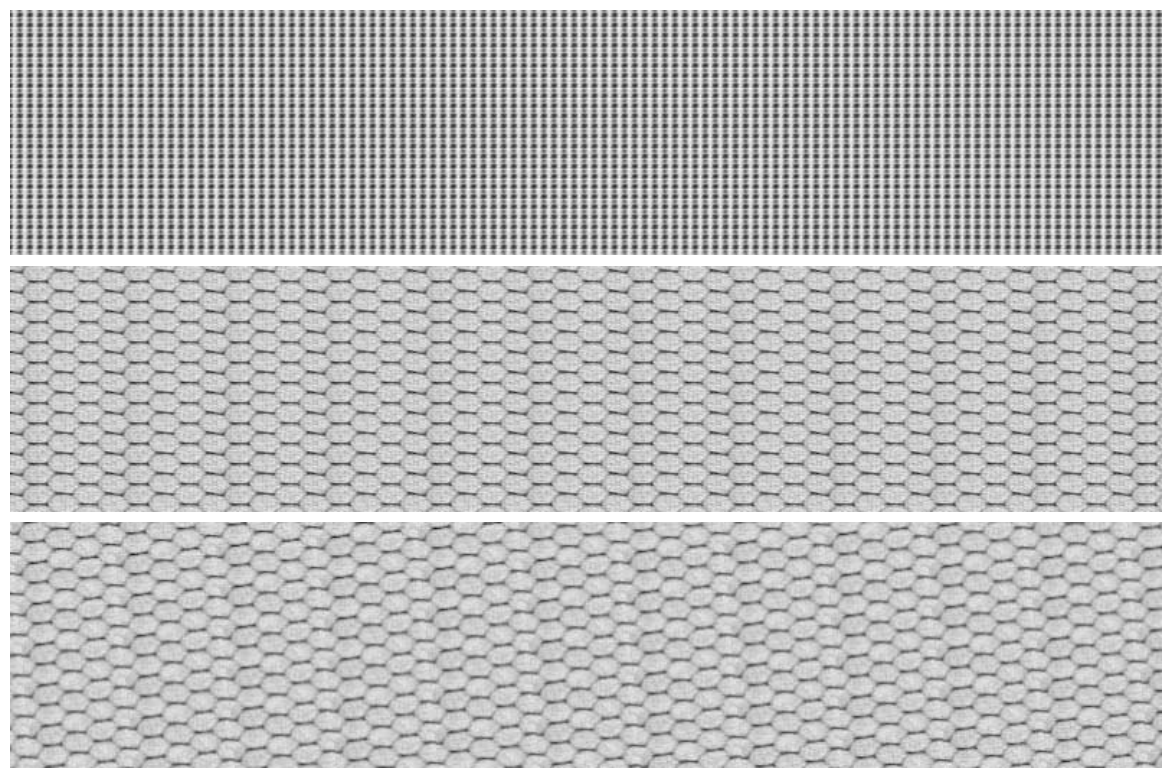

Fig. 3. Prototypes D1, D6, D14, D20, D21, and D34 (rotated $0^{\circ},-5^{\circ}$ ) 

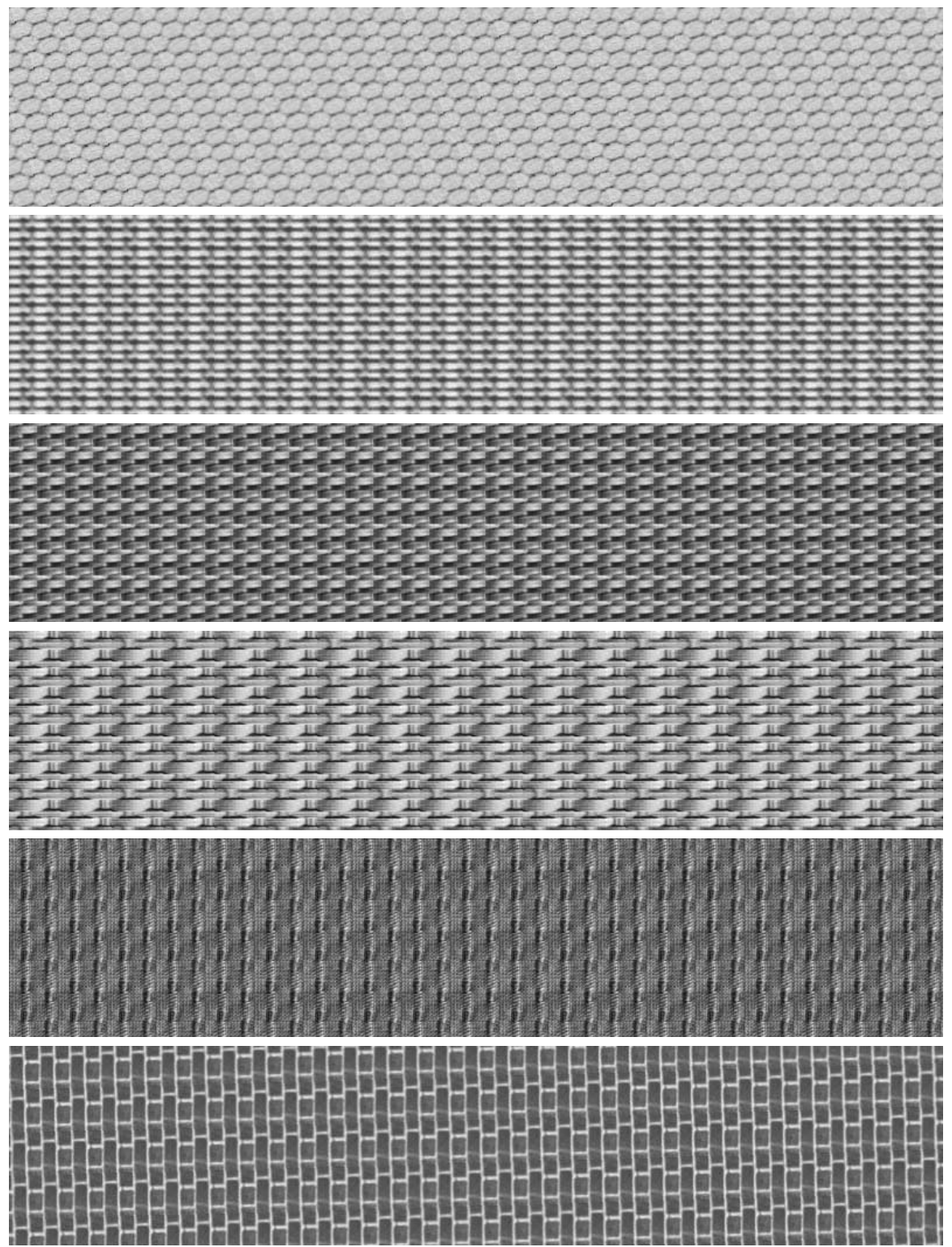

$\oiiint 0$

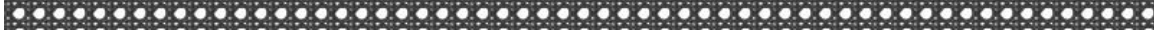

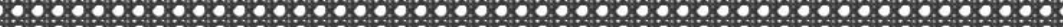

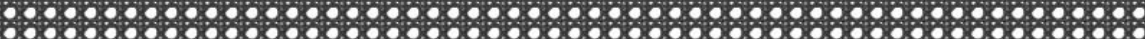

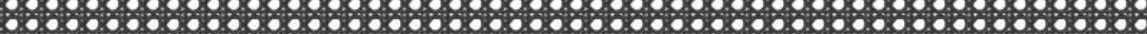

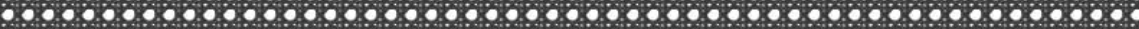

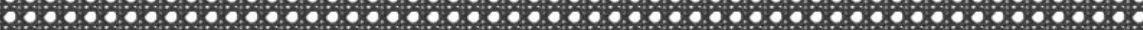

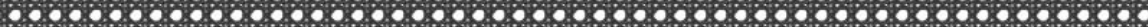

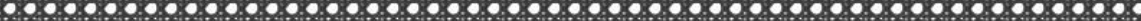

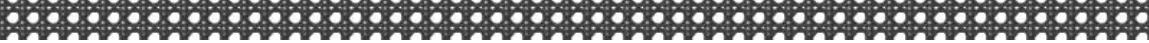

Fig. 4. Prototypes D34 (rotated -20²), D53, D55, D65, D83, D95, and D101 


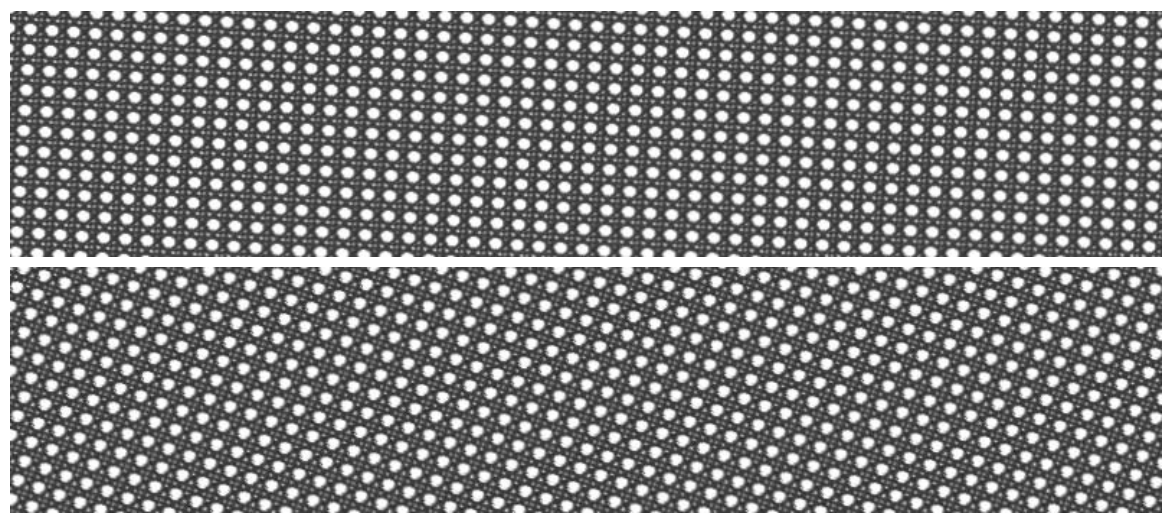

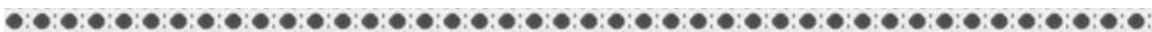

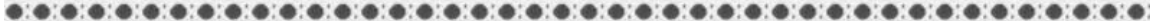

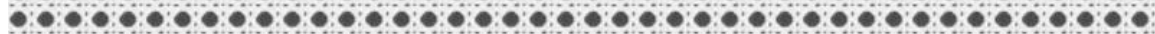

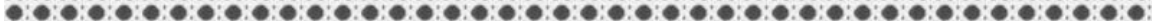

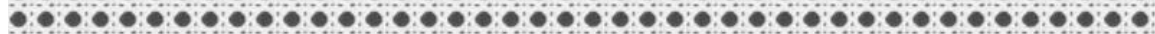

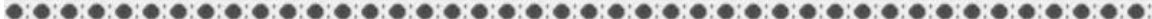

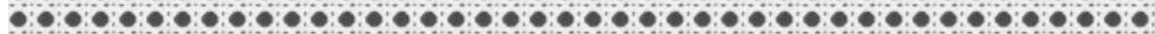

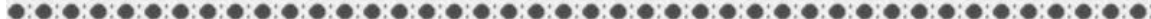

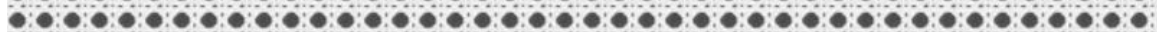

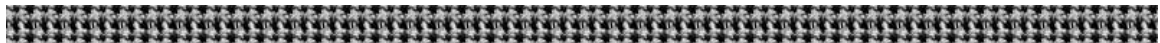

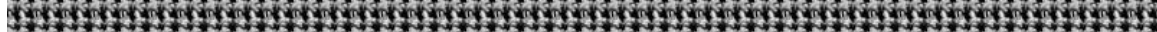

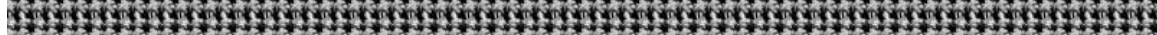

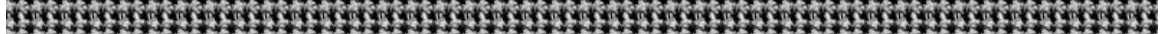

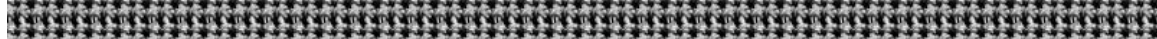
How

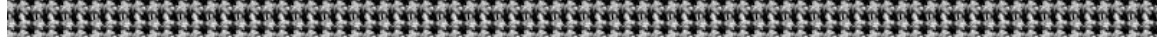

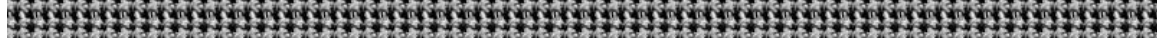
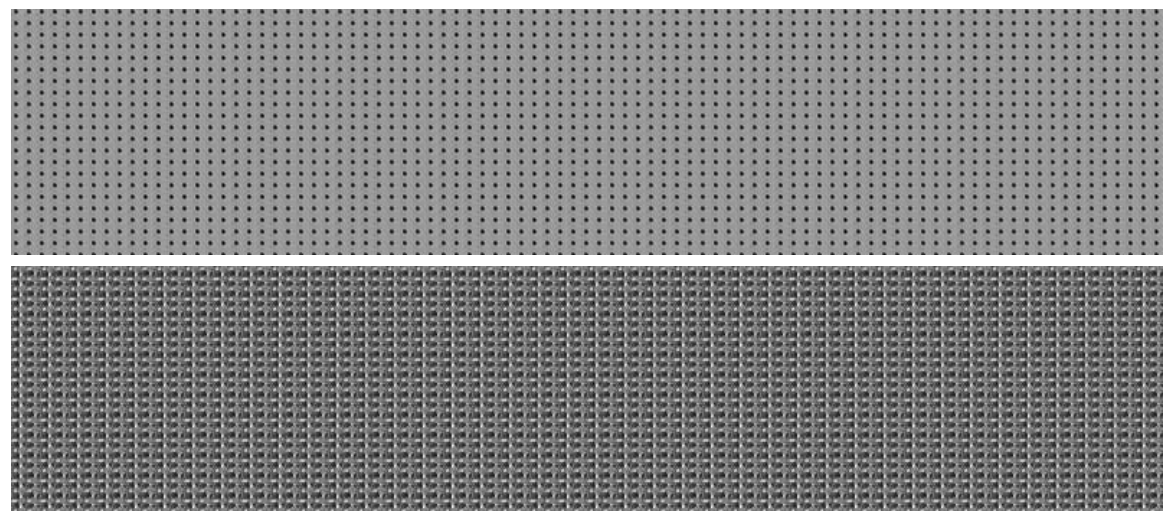

Fig. 5. Prototypes D101 (rotated $5^{\circ}, 20^{\circ}$ ), D102, Fabric0008, Tile0007, and Textile0025 
processed to exclude their relative distortions and produce an idealised texel. The texture prototype can easily be converted into a realistic sample by mutually agreed random photometric and geometric transformations of the adjacent tiles.

\section{Concluding Remarks}

Our experiments show that the orientation and size of a rectangular texel can be estimated from the structure of pairwise pixel interactions reflected in the MBIMs. Each tile obtained by partitioning the training sample can act as a provisional texel. But to obtain the ideal texel, the tiles should be jointly processed in order to exclude their geometric and photometric distortions. Replication of the texel forms a prototype of the texture.

The texel-based description is not adequate for all irregular (stochastic) textures with the MBIMs containing no repetitive peripheral energy clusters. But this description is practicable for many translation invariant regular mosaics. Because of computational simplicity of the texel estimation, the simulation of the large-size prototypes of such mosaics is considerably accelerated.

\section{References}

1. Brodatz, P.: Textures: A Photographic Album for Artists and Designers. Dover Publications: New York (1966) 178

2. De Bonet, J. S.: Multiresolution sampling procedure for analysis and synthesis of texture images. In: Proc. ACM Conf. Computer Graphics SIGGRAPH'97 (1997) 361-368 177

3. Efros, A. A., Leung, T. K.: Texture synthesis by non-parametric sampling. In: Proc. IEEE Int. Conf. Computer Vision ICCV'99, Greece, Corfu, Sept. 1999, vol.2 (1999) 1033-1038 177

4. Gimel'farb, G. L.: Image Textures and Gibbs Random Fields. Kluwer Academic: Dordrecht (1999) 177, 178

5. Gimel'farb, G.: Characteristic interaction structures in Gibbs texture modeling. In: Blanc-Talon, J., Popescu, D. C. (Eds.): Imaging and Vision Systems: Theory, Assessment and Applications. Nova Science: Huntington, N. Y. (2001) 71-90 177, 178

6. Haralick, R. M., Shapiro, L. G.: Computer and Robot Vision, vol.2. AddisonWesley: Reading (1993) 177

7. Liang, L., Liu, C., Xu, Y., Guo, B., Shum, H. Y.: Real-Time Texture Synthesis by Patch-Based Sampling. MSR-TR-2001-40. Microsoft Research (2001) 177, 178

8. Pickard, R., Graszyk, S., Mann, S., Wachman, J., Pickard, L., Campbell, L.: VisTex Database. MIT Media Lab.:Cambridge, Mass. (1995) 178

9. Paget, R., Longstaff, I. D.: Texture synthesis via a noncausal nonparametric multiscale Markov random field. IEEE Trans. on Image Processing 7 (1998) 925-931 177

10. Portilla, J., Simoncelli, E. P.: A parametric texture model based on joint statistics of complex wavelet coefficients. Int. Journal on Computer Vision 40 (2000) 49-71 177 
11. Zalesny, A., Van Gool, L.: A compact model for viewpoint dependent texture synthesis. In: Pollefeys, M., Van Gool, L., Zisserman, A., Fitzgibbon, A. (Eds.): 3D Structure from Images (Lecture Notes in Computer Science 2018). Springer: Berlin (2001) 124-143 177, 178 